\title{
Different corticostriatal integration in spiny projection neurons from direct and indirect pathways
}

\section{Edén Flores-Barrera, Bianca J. Vizcarra-Chacón, Dagoberto Tapia, José Bargas and Elvira Galarraga*}

División de Neurociencias, Instituto de Fisiología Celular, Universidad Nacional Autónoma de México, México City, México

\section{Edited by:}

Ranulfo Romo,

Universidad Nacional Autónoma de

México, México

\section{Reviewed by:}

José Manuel Giménez-Amaya,

Universidad Autónoma de Madrid,

Spain

Patricio O'Donnell,

University of Maryland School of

Medicine, USA

\section{*Correspondence:}

Elvira Galarraga, División de

Neurociencias, Instituto de Fisiología

Celular, Universidad Nacional

Autónoma de México, PO Box: 70-253,

México City, DF 04510 México.

e-mail:egalarra@ifc.unam.mx
The striatum is the principal input structure of the basal ganglia. Major glutamatergic afferents to the striatum come from the cerebral cortex and make monosynaptic contacts with medium spiny projection neurons (MSNs) and interneurons. Also: glutamatergic afferents to the striatum come from the thalamus. Despite differences in axonal projections, dopamine (DA) receptors expression and differences in excitability between MSNs from "direct" and "indirect" basal ganglia pathways, these neuronal classes have been thought as electrophysiologically very similar. Based on work with bacterial artificial chromosome (BAC) transgenic mice, here it is shown that corticostriatal responses in $D_{1}$ - and $D_{2}$-receptor expressing $M S N s\left(D_{1}\right.$ - and $\left.D_{2}-M S N s\right)$ are radically different so as to establish an electrophysiological footprint that readily differentiates between them. Experiments in BAC mice allowed us to predict, with high probability $(P>0.9)$, in rats or non-BAC mice, whether a recorded neuron, from rat or mouse, was going to be substance $P$ or enkephalin (ENK) immunoreactive. Responses are more prolonged and evoke more action potentials in $\mathrm{D}_{1}-\mathrm{MSN}$ s, while they are briefer and exhibit intrinsic autoregenerative responses in $\mathrm{D}_{2}-\mathrm{MSN}$ s. A main cause for these differences was the interaction of intrinsic properties with the inhibitory contribution in each response. Inhibition always depressed corticostriatal depolarization in $\mathrm{D}_{2}-\mathrm{MSNs}$, while it helped in sustaining prolonged depolarizations in $\mathrm{D}_{1}-\mathrm{MSNs}$, in spite of depressing early discharge. Corticostriatal responses changed dramatically after striatal DA depletion in 6-hydroxy-dopamine (6-OHDA) lesioned animals: a response reduction was seen in substance $\mathrm{P}(\mathrm{SP})+\mathrm{MSN}$ whereas an enhanced response was seen in ENK+ MSNs. The end result was that differences in the responses were greatly diminished after DA depletion.

Keywords: basal ganglia, strionigral pathway, striopallidal pathway, medium spiny neurons, GABA $_{A}$ receptors, corticostriatal pathway, 6-hydroxy-dopamine, Parkinson disease

\section{INTRODUCTION}

Models of basal ganglia function are commonly based on the segregation of medium spiny projection neurons (MSNs) into "direct" and "indirect" pathways which act in opposite ways to control movement (DeLong, 1990; Obeso et al., 2004). GABAergic neurons of the direct strionigral pathway are characterized by a higher expression of $\mathrm{D}_{1}$ dopamine receptors plus the expression of substance $\mathrm{P}(\mathrm{SP})$ and dynorphin ( $\mathrm{D}_{1}$-MSNs), whereas GABAergic neurons of the indirect striopallidal pathway are characterized by a higher expression of $\mathrm{D}_{2}$ dopamine receptors and co-expression of enkephalins ( $\mathrm{D}_{2}$-MSNs) (Gerfen and Young, 1988). However, a robust way to differentiate these neurons electrophysiologically has not been described, although differences in their latency for initial firing, threshold, subtype of inward rectification, frequency of spontaneous events, their capacity to develop long term synaptic plasticity after corticostriatal stimulation, and the subunit composition of their GABAergic receptors have been reported (Kreitzer and Malenka, 2007; Shen et al., 2007; Ade et al., 2008; Cepeda et al., 2008; Day et al., 2008; Valjent et al., 2009). Unfortunately, none of these differences can be readily used to identify the pathway, direct or indirect, to which a recorded MSN belongs.

The present experimental work shows that suprathreshold corticostriatal responses are an electrophysiological footprint that serves to recognize whether a recorded MSN belongs to the direct or indirect pathway with high probability. More importantly, these differences disclose different integration processes for striatal inhibitory inputs.

Major glutamatergic afferents to the striatum come from the cerebral cortex and are monosynaptic to both MSNs and interneurons (e.g., Kawaguchi et al., 1995). Suprathreshold corticostriatal responses may exhibit plateau depolarizations lasting hundreds of milliseconds capable to fire trains of action potentials (Bargas et al., 1991; Kita, 1996). These orthodromic responses include polysynaptic or reverberating inputs as well as intrinsic autoregenerative responses (Wilson, 1986; Kita, 1996; O’Donnell, 2003; Vergara et al., 2003; Tseng et al., 2007; Flores-Barrera et al., 2009) and are the first step for micro-circuitry integration and processing (Carrillo-Reid et al., 2008, 2009a). The present work demonstrates with the help of bacterial artificial chromosome (BAC) transgenic mice expressing enhanced green fluorescent protein (eGFP) under the control of $\mathrm{D}_{1}$ or $\mathrm{D}_{2}$ receptor promoters (BAC $D_{1}$ or $\mathrm{D}_{2}$ eGFP mice), large, distinct and clear differences between the corticostriatal responses from direct and indirect pathway neurons. Moreover, these electrophysiological differences allowed us to predict, with high probability $(P>0.9)$, whether a recorded neuron, from rat or non-BAC mice, was going to react to SP or enkephalin (ENK) antibodies. Thereafter, we were able to demonstrate that these differences are greatly altered in 
neurons from dopamine (DA)-depleted striata after unilateral 6-hydroxy-dopamine (6-OHDA) lesions in the subtantia nigra compacta $(\mathrm{SNc})$.

\section{MATERIALS AND METHODS SLICE PREPARATION}

All experiments were carried out in accordance with the National Institutes of Health Guide for Care and Use of Laboratory Animals and were approved by the Institutional Animal Care Committee of the Universidad Nacional Autonoma de Mexico. BAC $\mathrm{D}_{1}$ and $\mathrm{D}_{2}$ eGFP mice, between postnatal days 60-90 (PD60-90) or adult Wistar rats (PD90) were used. Animals were anesthetized with ketamine/xylazine and their brains quickly removed and placed into ice cold $\left(4^{\circ} \mathrm{C}\right.$ ) bath saline containing (in $\mathrm{mM}$ ): $126 \mathrm{NaCl}, 3 \mathrm{KCl}$, $25 \mathrm{NaHCO}_{3}, 2 \mathrm{MgCl}_{2}, 2 \mathrm{CaCl}_{2}, 11$ glucose, $300 \mathrm{mOsm} / \mathrm{l}, \mathrm{pH}=7.4$ gassed with $95 \% \mathrm{O}_{2}$ and $5 \% \mathrm{CO}_{2}$. Parasagittal neostriatal slices (250-350 $\mu \mathrm{m}$ thick) were cut using a vibratome and incubated in oxygenated bath saline at room temperature for at least $1 \mathrm{~h}$ before recording.

\section{INTRACELLULAR RECORDINGS}

Intracellular recordings were carried out using sharp microelectrodes (80-120 M $\Omega$ ) filled with $1 \%$ biocytin and $3 \mathrm{M}$ potassium acetate fabricated from borosilicate glass. Recordings were obtained with a high input impedance electrometer (Neurodata, New York, NY, USA) with an active bridge circuit using standard techniques (see Flores-Barrera et al., 2009). Signals were filtered at $1-3 \mathrm{kHz}$ and digitized at $3-9 \mathrm{kHz}$ with an AT-MIO-16E 4 board (National Instruments, Austin, TX, USA) and a PC computer. Slices were totally submerged in the bath solution and superfused with the same saline at $2 \mathrm{ml} / \mathrm{min}\left(34-36^{\circ} \mathrm{C}\right)$. Recordings were digitized and captured with the aid of software designed in the laboratory in the LabView environment (National Instrument, Austin, TX, USA) (Lemus-Aguilar et al., 2006). Resting membrane potential was about $-83 \pm 4 \mathrm{mV}$; whole neuronal input resistance $\left(R_{\mathrm{N}}\right)$ was about $48 \pm 6 \mathrm{M} \Omega$, membrane time constant $\left(\tau_{\mathrm{m}}\right)$ was about $33 \pm 6 \mathrm{~ms}$ and equalizing time constant $\left(\tau_{1}\right)$ was about $5 \pm 0.8 \mathrm{~ms}(n=46)$ as measured by fitting a sum of exponential functions to the voltage transients (see also Reyes et al., 1998). No significant differences in passive properties were found between indirect and direct pathway neurons, neither between control and DA-depleted neurons (Cepeda et al., 2008; Gertler et al., 2008). Drugs were dissolved in the bath saline from stock solutions made daily.

\section{EVOKED CORTICOSTRIATAL RESPONSES}

Recordings were carried out in the dorsal striatum. The arrangement of recording and stimulating electrodes has been described (see Flores-Barrera et al., 2009). Briefly, the stimulation of cortical sensory-motor areas was performed with concentric bipolar electrodes $(25-50 \mu \mathrm{m}$ at the tip). The distance between recording and stimulating electrodes was between 0.5 and $1 \mathrm{~mm}$. Synaptic responses were evoked by a single unrepeated square wave pulse of $0.1 \mathrm{~ms}$. Stimulation was delivered with a stimulator (S-8800; Grass, West Warwick, RI, USA) using an isolation unit. The membrane potential was held at $-80 \mathrm{mV}$ while the corticostriatal responses were recorded. A series of current pulses of increasing intensity were used to determine evoked synaptic potential threshold and response amplitude at different stimulation strengths. A series of stimulus $(0.5 \times, 0.75 \times, 1.25 \times, 1.5 \times, 2.0 \times$ threshold current) was used to compare response amplitudes, as well as the effects of drugs used. Traces shown are the average of three recordings taken after the amplitude had been stabilized in a given condition.

\section{CELL VISUALIZATION AND CONFIRMATION OF CELL IDENTITY}

Medium spiny projection neurons within the dorsal striatum were identified by their enhanced expression of green fluorescent protein (eGFP) using epifluorescence microscopy (dopamine $\mathrm{D}_{1}$ or $\mathrm{D}_{2}$ receptor expression). In all cases neurons were injected with biocytin and sub-samples of neurons were immunoreacted to either SP or ENK antibodies. Inmmunocytochemical preparations were visualized on a confocal microscope (see Guzman et al., 2003). Data obtained from BAC $D_{1}$ or BAC $D_{2}$ eGFP mice were compared. Because electrophysiological responses from eGFP negative neurons from $B A C D_{1}$ eGFP cannot be discerned from those recorded from eGFP-positive neurons from $B A C D_{2}$ eGFP mice, and vice versa, their measurements of corticostriatal responses were pooled together. Moreover, corticostriatal responses identified as coming from direct or indirect pathway neurons were also identified in rat neurons. In fact, we calculated the probability to ascertain the proper identity of a rat neuron by predicting, according to its corticostriatal response, its correspondent pathway (direct or indirect) as established by immunocytochemistry for SP or ENK. This probability was $>0.9$. This allowed us to use the hemiparkinsonian rat model of Parkinson disease (6-hydroxy-dopamine or 6-OHDA) to test whether corticostriatal responses changed as a result of DA depletion.

\section{6-OHDA LESIONS}

Procedures to deplete the striatum of DA after 6-OHDA in the SNc have been described (Dunnett et al., 1981). Briefly, male Wistar rats (P60-90) were anesthetized with ketamine $(85 \mathrm{mg} / \mathrm{kg}$, i.p.) and xylazine $(15 \mathrm{mg} / \mathrm{kg}, \mathrm{i} . \mathrm{p})$ and injected stereotaxically with $4 \mu \mathrm{g}$ of 6-OHDA (Sigma, St. Louis, MO, USA) into the left substantia nigra (SN: $4.80 \mathrm{~mm}$ caudal, $1.6 \mathrm{~mm}$ lateral to bregma, and $8.2 \mathrm{~mm}$ ventral to the skull surface). 6 -OHDA solution $(4 \mu \mathrm{g} / \mu \mathrm{l}$ saline with $0.2 \mathrm{mg} / \mathrm{ml}$ of ascorbic acid) was ejected at a rate of $0.1 \mu \mathrm{l} / \mathrm{min}$. After 2 weeks animals were selected based on their rotational behavior (amphetamine: $4 \mathrm{mg} / \mathrm{kg}$ i.p.; >500 ipsilateral stereotyped turns) (Inaji et al., 2005). The number of animals used in the experimental samples was the minimal possible to attain statistical significance under diverse experimental paradigms. All procedures employed anesthesia to avoid animal suffering.

\section{DATA ANALYSIS AND STATISTICS}

The area under the corticostriatal synaptic response was obtained by numerical integration and then normalized to the area under the threshold voltage (Flores-Barrera et al., 2009). Percentages of change in this area are reported. Digital subtraction was also used to obtain the component sensitive to bicuculline, $\mathrm{a} \mathrm{GABA}_{\mathrm{A}}$ receptor antagonist. Statistical values in histograms and text are presented as mean \pm SEM. Differences between measured parameters were compared with Student's $t$-tests. 


\section{RESULTS}

\section{$D_{1}$ AND $D_{2}$ MEDIUM SPINY NEURONS DIFFERED IN THEIR SUPRATHRESHOLD CORTICOSTRIATAL RESPONSES}

The MSNs presented in this study $(n=200)$ were identified as belonging to the direct pathway ( $\mathrm{D}_{1}-\mathrm{SP}+\mathrm{MSN}$ ) based on: first, the expression of dopamine $\mathrm{D}_{1}$ receptor identified by their expression of GFP (from PD60-90 BAC mice), and second, by their SP-immunoreactivity (SP+; from mice or rats). A similar procedure was done to identify indirect pathway neurons ( $\left.\mathrm{D}_{2}-\mathrm{ENK}+\mathrm{MSNs}\right)$ : $\mathrm{D}_{2}$ eGFP (from BAC mice) and ENK-immunoreactivity (ENK+; from mice or rat) (see Materials and Methods). Figure 1 shows corticostriatal responses from a typical BAC $D_{1}$ eGFP MSN $\left(\mathrm{D}_{1}-\mathrm{MSN}\right)\left(\right.$ Figure 1A) and a typical BAC $\mathrm{D}_{2}$ eGFP MSN $\left(\mathrm{D}_{2}-\mathrm{MSN}\right)$ (Figure 1B). Families of subthreshold and suprathreshold corticostriatal responses for these two neuron classes are illustrated. A large, conspicuous and evident difference between these responses
A

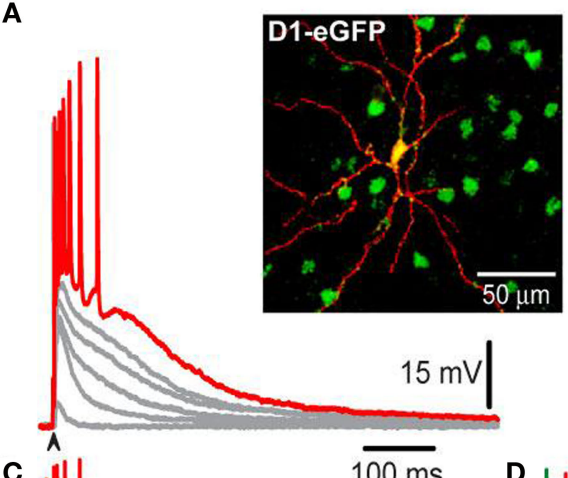

c
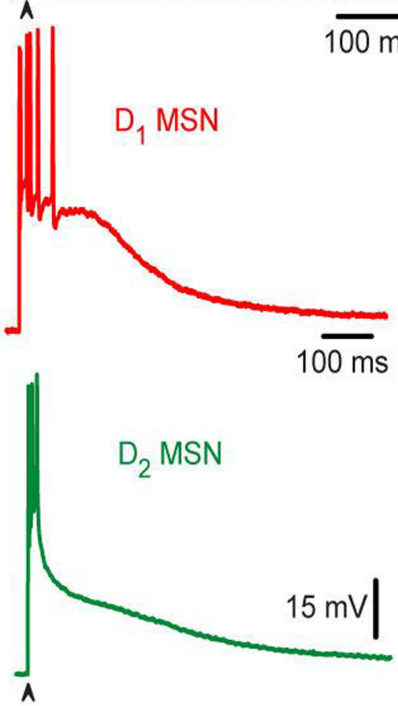

$\mathbf{F}$

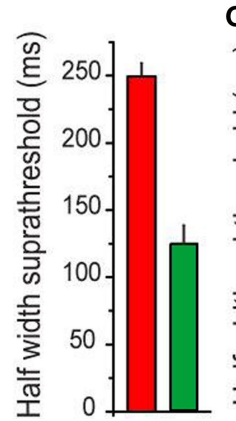

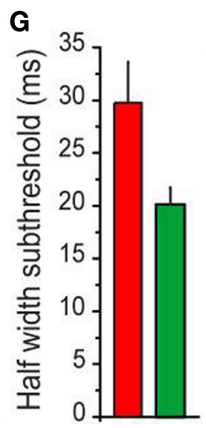
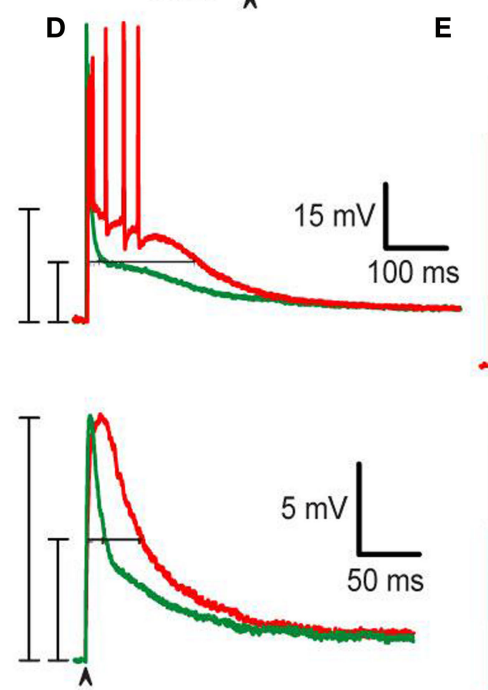

$\mathbf{H}$
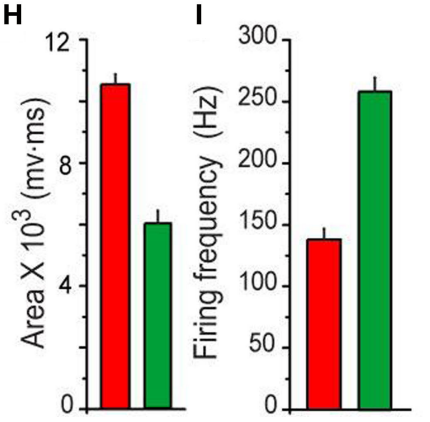

B

FIGURE 1 | Different corticostriatal response in $D_{1}$ or $D_{2}$ eGFP medium spiny neurons (MSNs) from BAC mice. (A,B) Families of subthreshold and suprathreshold corticostriatal synaptic responses for representative $D_{1}$ - (red traces) and $\mathrm{D}_{2}$ - (green traces) MSNs obtained from BAC transgenic mice striatum. Insets show biocytin-filled recorded cells and green fluorescent protein positive (GFP+) accompanying cells (insets). Yellow color of recorded cells indicate $D_{1}-(A)$ and $D_{2}$-eGFP-positive cells (B), respectively (superimposition). Notice that $\mathrm{D}_{1}-\mathrm{MSN}$ s suprathreshold response is more prolonged and generates more action potentials whereas $\mathrm{D}_{2}-\mathrm{MSNS}$ response only display an initial burst of high frequency firing. (C,D) Area under response is smaller in $D_{2}-M S N s$, as well as half-widths (F-H) Histograms summarize measurements in neuronal samples (half-widths and areas under responses): $n=55 \mathrm{D}_{1}-\mathrm{MSNs}$ and $n=50$ $D_{2}-M S N s$. Differences were significant. (E) Traces at a slower sweep (1-3) illustrate frequency of discharge in $D_{1}-(1), D_{2}-M S N s$ (2) as well as intrinsic autoregenerative responses in $\mathrm{D}_{2}-\mathrm{MSN}$ s. (I) Firing frequency was higher in $\mathrm{D}_{2}$-MSNs. 
can be seen at a first glance: Responses of $\mathrm{D}_{1}$-MSNs last longer than those from $\mathrm{D}_{2}$-MSNs, including both subthreshold depolarizations (gray) and suprathreshold depolarizations with trains of action potentials (colored) (Figure 1C cf., top vs. bottom). The same results were obtained in neurons from rats predicted to be SP+ or ENK+ according to the same electrophysiological responses (see Materials and Methods). Mean durations of suprathreshold responses at half amplitude (half-widths) were: $250 \pm 9 \mathrm{~ms}$ for $\mathrm{D}_{1}-\mathrm{SP}+\mathrm{MSNs}$ $(n=55)$ and $124 \pm 13 \mathrm{~ms}$ for $\mathrm{D}_{2}-\mathrm{ENK}+\mathrm{MSNs}(n=50)$ (Figure 1D top and histogram in Figure 1F; $P<0.001)$. Mean half-widths of subthreshold responses were also different: $29 \pm 3 \mathrm{~ms}$ for $\mathrm{D}_{1}-\mathrm{SP}+$ MSNs and $19 \pm 2 \mathrm{~ms}$ for $\mathrm{D}_{2}$-ENK+ MSNs (Figure 1D bottom and histogram in Figure 1G; $P<0.005)$. Moreover, areas under corticostriatal responses were significantly larger in $\mathrm{D}_{1}-\mathrm{SP}+\mathrm{MSN}$ than in $\mathrm{D}_{2}$-ENK+ MSNs: $10,546 \pm 331 \mathrm{mV} \cdot \mathrm{ms}$ vs. 5,987 $\pm 427 \mathrm{mV} \cdot \mathrm{ms}$, respectively $(P<0.001$; histogram in Figure $\mathbf{1 H})$. Figures 1E1,E2 illustrate the same responses at a slower sweep: trains of action potentials in $\mathrm{D}_{1}-\mathrm{SP}+\mathrm{MSN}$ lasted more but attained a slower mean firing frequency than trains in $\mathrm{D}_{2}$-ENK+ MSNs: $138 \pm 8 \mathrm{~Hz}$ vs. $258 \pm 11 \mathrm{~Hz}$, respectively $(P<0.001$, histogram in Figure 1I). Finally, in the case of $\mathrm{D}_{2}$-ENK+ MSNs, suprathreshold responses frequently exhibit intrinsic autoregenerative potentials instead of the brief train of spikes (in some trials during the same experiment) (Figure 1E3), suggesting that this intrinsic property underlies the train of action potentials and shapes the response in $\mathrm{D}_{2}$-ENK+ MSNs (Bargas et al., 1991; Kita, 1996; Gruber et al., 2003; Vergara et al., 2003; Carter and Sabatini, 2004; Day et al., 2008; Flores-Barrera et al., 2009). These responses were rarely seen in $D_{1}-S P+$ MSNs in these conditions, but were easily disclosed in all $\mathrm{D}_{2}$-ENK+ MSNs when subject to hyperpolarization (not shown here but see Bargas et al., 1991), supporting previous suggestions that dendritic excitability, and therefore, corticostriatal integration is different in $\mathrm{D}_{1}-\mathrm{SP}+$ and $\mathrm{D}_{2}$-ENK+ MSNs (Cepeda et al., 2008; Day et al., 2008).

\section{CONTRIBUTION OF SYNAPTIC INHIBITION DIFFERED IN THE CORTICOSTRIATAL RESPONSES OF DIRECT AND INDIRECT PATHWAY NEURONS}

It is known that inhibitory inputs from axon collaterals interconnecting MSNs (Tunstall et al., 2002; Guzman et al., 2003; Koos et al., 2004; Tecuapetla et al., 2005, 2007, 2009; Gustafson et al., 2006; Gertler et al., 2008) and from several types of GABAergic interneurons (Koos et al., 2004; Tecuapetla et al., 2007) contribute to suprathreshold corticostriatal responses (Flores-Barrera et al., 2009). Some differences in the actions of GABAergic inputs onto $\mathrm{D}_{1}$ - and $\mathrm{D}_{2}$-MSNs have been shown (Ade et al., 2008; Cepeda et al., 2008; Tecuapetla et al., 2009; Gittis et al., 2010; Planert et al., 2010). However, the probable contribution of these differences in the shaping of corticostriatal responses is not known. Figure 2 shows that important functional differences can be disclosed for the contribution of inhibition on the suprathreshold responses from $\mathrm{D}_{1}-\mathrm{SP}+$ and $\mathrm{D}_{2}$-ENK+ MSNs. Representative examples of orthodromic responses from $\mathrm{D}_{1}-\mathrm{SP}+$ and $\mathrm{D}_{2}$-ENK+ MSNs are shown in Figures 2A,E (control). Figures 2B,F show that the action of the $\mathrm{GABA}_{\mathrm{A}}$ receptor blocker, $10 \mu \mathrm{M}$ bicuculline, is completely different for each response: $\mathrm{GABA}_{\mathrm{A}}$ receptor blockade in $\mathrm{D}_{1}-\mathrm{SP}+\mathrm{MSN}(n=24)$ had a dual action (Figure 2B): Early in the response it produced an enhancement of the initial depolarization and an increase in the frequency of evoked discharge, suggesting that initial depolarization and firing are being restrained by the GABAergic inputs contributing to the response (Flores-Barrera et al., 2009). However, later in the response the plateau depolarization was reduced by GABA blockade, indicating that GABAergic inputs help in sustaining the depolarization (depolarizing inhibition) (Gulledge and Stuart, 2003; Bartos et al., 2007; Mann and Paulsen, 2007; Flores-Barrera et al., 2009).

Superimposition (Figure 2C: superimposition of Figures 2A,B) and subtraction of recordings (Figure 2D: subtraction of Figures 2A,B), disclose the dual action of GABA in direct pathway neurons: the bicuculline-sensitive component first hyperpolarizes the neuron with respect to firing level, and second, depolarizes the neuron and helps in sustaining the depolarization late in the response: superimposed traces cross each other (Figure 2C). Percentages of early depolarization (before crossing) and late hyperpolarization (after crossing) induced by bicuculline in the synaptic response are summarized in the histogram at the inset of Figure 2D: $28 \pm 4 \%$ (early) and $-25 \pm 3 \%$ (late) $(P<0.002)$.

In contrast, bicuculline behaved differently in the indirect pathway MSNs responses (cf., Figures 2E,F): it depolarized the response all along. Superimposition of recordings, before and after bicuculline (Figure 2G), do not cross each other. And subtraction of them (Figures 2E,F,H) discloses a bicuculline-sensitive component whose action persisted during the whole trace; that is, inhibition decreases the amplitude of the response at all times. Percentage of amplitude depolarization increase after bicuculline was $76 \pm 10 \%$ (inset of Figure 2D; $n=22 ; P<0.002$ ) in $\mathrm{D}_{2}$-ENK+ MSNs responses. The number of action potentials and duration of the train also increased. However, some spikes inactivated due to the amount of depolarization attained in $\mathrm{D}_{2}$-ENK+ MSNs after bicuculline. This large depolarization reflects the underlying intrinsic autoregenerative response below the train of action potentials in control responses from $\mathrm{D}_{2}$-ENK+ MSNs (Bargas et al., 1991; Carrillo-Reid et al., 2009a,b) which is facilitated by bicuculline (Figure 1E3). Therefore, the result strongly suggests that a main role of inhibition in $\mathrm{D}_{2}$-ENK+ MSNs is to restrain intrinsic dendritic excitability (Figure 2G) (Bargas et al., 1991; Carter and Sabatini, 2004; Day et al., 2008). Histogram at the inset of Figure $\mathbf{2} \mathbf{H}$ compares half-widths of responses from each cell class after bicuculline, with respect to their own controls (dashed line): clearly, $\mathrm{D}_{1}-\mathrm{SP}+\mathrm{MSNs}$ response decrease in duration (ca. $76 \% ; 249 \pm 9 \mathrm{~ms}$ to $189 \pm 2 \mathrm{~ms}$ ) while $\mathrm{D}_{2}$-ENK+ MSNs response increase in duration (ca. 192\%; $124 \pm 1 \mathrm{~ms}$ to $238 \pm 8 \mathrm{~ms}$ ) during bicuculline action. Both picrotoxin and gabazine had the same actions as bicuculline (not shown).

We conclude that the role of inhibition is different in the corticostriatal suprathreshold responses of direct and indirect pathways MSNs. In direct neurons, inhibition restrains initial firing but helps in maintaining plateau depolarization. In indirect pathway neurons, inhibition has the function of suppressing intrinsic excitability at dendritic levels and thus firing of action potentials (Bargas et al., 1991; Cepeda et al., 2008; Day et al., 2008; FloresBarrera et al., 2009).

If the above conclusion is correct we should be able to distinguish different roles of inhibition in subthreshold responses also, because at lower stimulus strengths we may be able to record depolarizing non-propagating intrinsic responses (Carter 
A

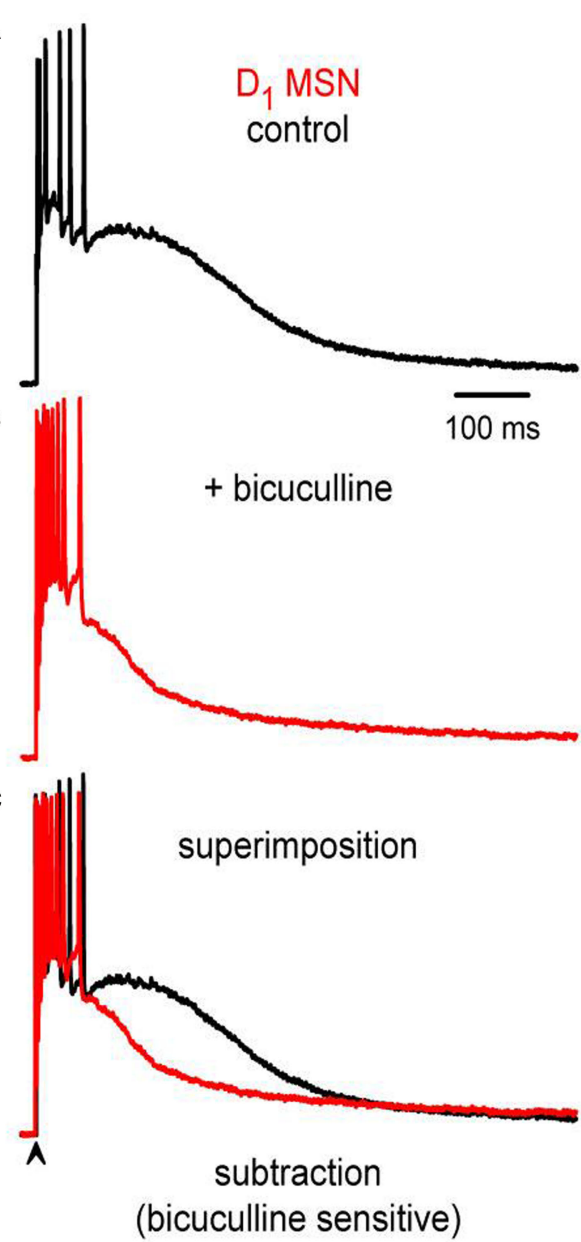

D

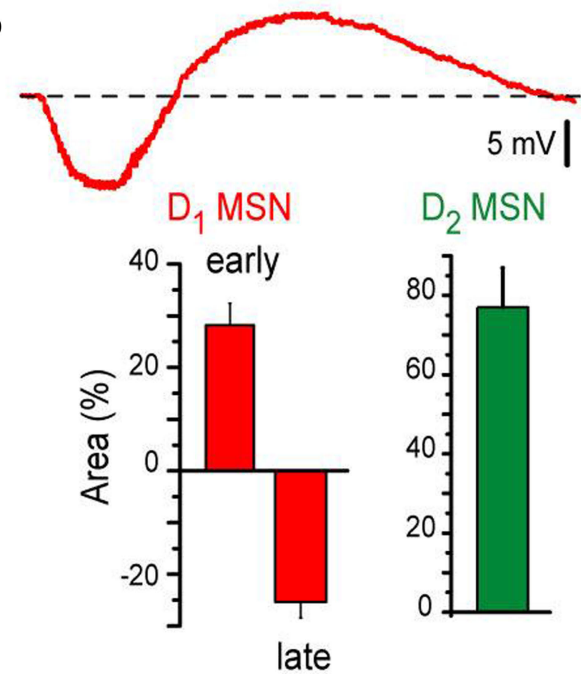

FIGURE 2 | Contribution of synaptic inhibition differs in corticostriatal responses from $D_{1}$ - and $D_{2}-M S N s$ from $B A C$ mice. $(A, E)$ Suprathreshold corticostriatal responses in $\mathrm{D}_{1}$ - and $\mathrm{D}_{2}-\mathrm{MSNs}$, respectively. (B,F) Addition of $10 \mu \mathrm{M}$ bicuculline to the bath saline (colored traces) changes both responses indicating that GABAergic events participate in each of them. $(\mathbf{C}, \mathbf{G})$ Superimposed records: (A) with (B) and (E) with (F), show that GABAergic blockade mostly reduce $D_{1}-M S N s$ response and enhance $D_{2}-M S N$ s response, respectively. Notice prolongation of action potentials trains in $\mathrm{D}_{2}-\mathrm{MSNs}$ with inactivation of some

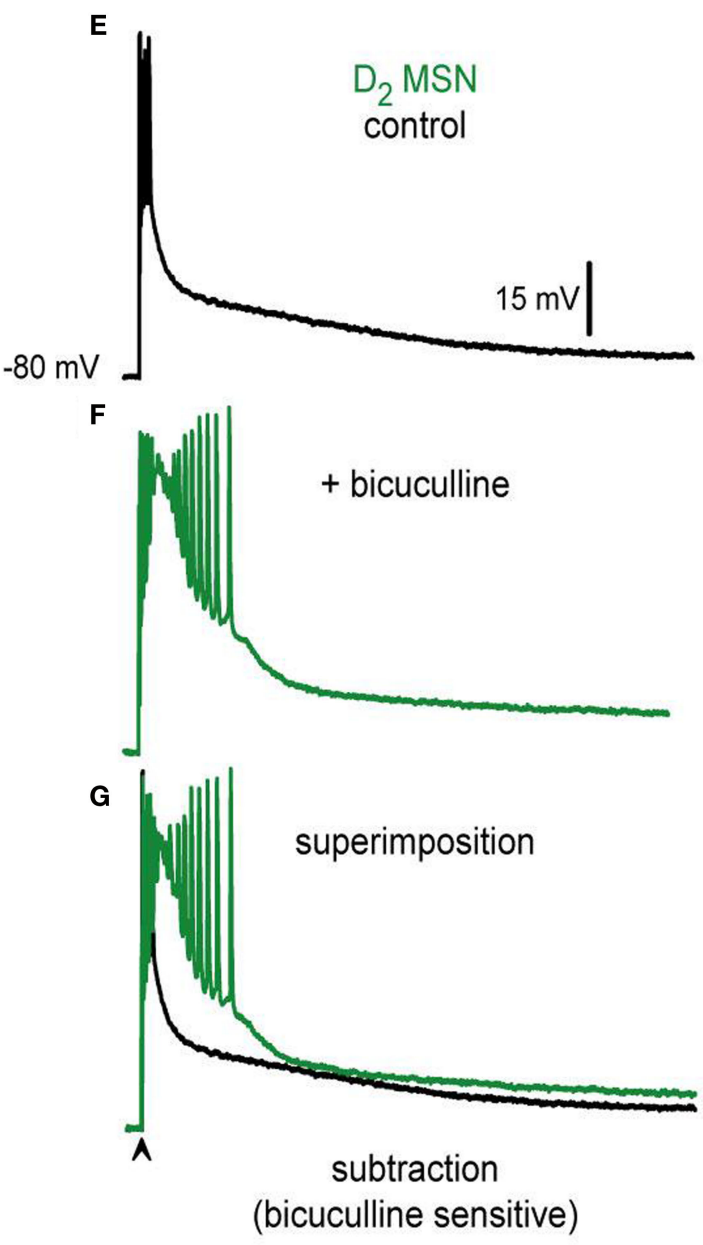

$\mathbf{H}$

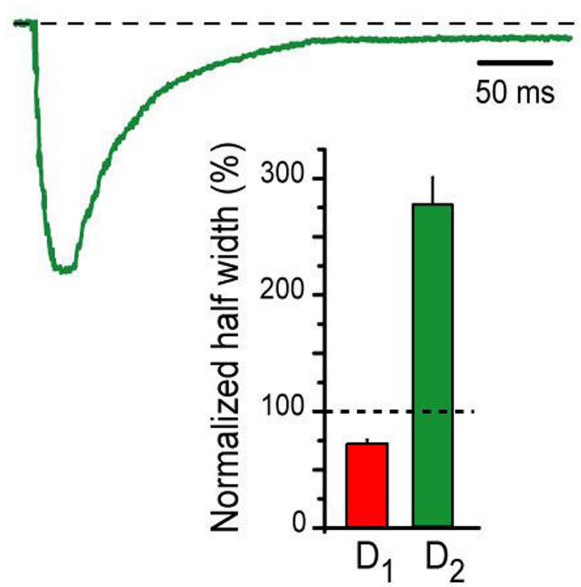

spikes. (D,H) Digital subtraction of records in (C), (G) disclose the bicucullinesensitive components in both neuron classes. Notice that except for an initial restrain in evoked discharge, the bicuculline-sensitive component depolarizes $\mathrm{D}_{1}$-MSNs. Insets: histograms show that bicuculline first enhances, then depresses $D_{1}-M S N$ s response (a biphasic response, red bars, $n=24$ ). In contrast, bicuculline only augments $D_{2}-M S N s$ response (green bar, $n=22$ ). In the same way, half-widths were decreased and enhanced for $D_{1}$ - and $\mathrm{D}_{2} \mathrm{MSNs}$, respectively. 
and Sabatini, 2004; Day et al., 2008). To test this hypothesis, the contribution of the bicuculline-sensitive component to the corticostriatal responses of direct and indirect pathway neurons was further observed at different stimulation intensities. The results are shown in Figure 3 (subthreshold, threshold and suprathreshold intensities): at subthreshold levels (cf., Figures 3A,E) bicuculline is revealed to depress synaptic inputs in $\mathrm{D}_{1}-\mathrm{SP}+\mathrm{MSN}$ s while it enhances synaptic inputs in $\mathrm{D}_{2}$-ENK+ MSNs. Note that control subthreshold synaptic events (black traces in Figures 3A,E) were depolarizing for both synaptic potentials near $10 \mathrm{mV}$ above rest, with no indication of a hyperpolarizing component, indicating that inhibitory contribution to these potentials is depolarizing for both types of neuron (i.e., reversal potentials less negative than resting potentials near $-80 \mathrm{mV}$ ). This has been corroborated by several studies using different techniques (Misgeld et al., 1982; Jiang and North, 1991; Bennett and Bolam, 1994; Kita, 1996; Ramanathan et al., 2002; Koos et al., 2004; Bracci and Panzeri, 2006; Dehorter et al., 2009; Flores-Barrera et al., 2009) discarding biases towards one neuronal class. Nonetheless, blockade of $\mathrm{GABA}_{\mathrm{A}}$ receptors during subthreshold responses decreases the synaptic potential in $\mathrm{D}_{1}-\mathrm{SP}+\mathrm{MSN}$ since a depolarizing component: inhibition, was blocked (colored trace in Figure 3A). In contrast, bicuculline enhances subthreshold responses in $\mathrm{D}_{2}$-ENK+ MSNs (Figure 3E colored trace) even if inhibition is also depolarizing. To explain how a depolarizing input enhances depolarization when it is blocked, one has to assume that inhibition in $\mathrm{D}_{2}$-ENK+ MSNs is restraining intrinsic depolarizing currents (shunting inhibition) even at subthreshold levels (Carter and Sabatini, 2004; Day et al., 2008) supporting the assumption made above.

This assumption is further supported by analyzing threshold inputs (cf., Figures 3B,F; spikes are clipped).

Noticeably, GABAergic synapses help to fire an action potential in $\mathrm{D}_{1}-\mathrm{SP}+\mathrm{MSN}$ since bicuculline avoided firing (Figure 3B colored trace); inhibition being both depolarizing and excitatory for these neurons at this stimulus strength (Vida et al., 2006). In contrast, bicuculline discloses an intrinsic local response during threshold inputs in $\mathrm{D}_{2}$-ENK+ MSNs (Figure 3F colored trace). Local response disrupts both kinetics and duration of synaptic potentials and preceded the appearance of intrinsic autoregenerative responses at stronger stimulus strengths (Figure 3G). Finally, Figures 3C-H illustrate suprathreshold traces and subtractions of the different paired traces (Figures 3A-G).

To summarize, although the bicuculline-sensitive component (i.e., GABA inputs) is depolarizing at all intensities in both types of MSNs and it actually contributes to synaptic depolarization and even firing in $\mathrm{D}_{1}$-SP+MSNs (Bracci and Panzeri, 2006), in $\mathrm{D}_{2}$-ENK+ MSNs it has another role, that of restraining intrinsic dendritic excitability (Day et al., 2008). These results demonstrate different roles for inhibition in direct and indirect pathway neurons. The cause of these different inhibitory roles may not be completely attributed to different inhibitory inputs (Mallet et al., 2006; Gittis et al., 2010; Planert et al., 2010), which is under debate, but to differences in intrinsic dendritic excitability of direct and indirect neurons (Day et al., 2008).

Finally, evidence has been shown that a tonic current mediated by $\mathrm{GABA}_{\mathrm{A}}$ receptors containing the $\alpha_{5}$ subunit is more expressed in the $D_{2}$ - than in the $D_{1}$-MSNs (Ade et al., 2008). Therefore, we wanted to see the contribution of this current to the difference between the responses. $10 \mu \mathrm{M}$ L655-708, an inverse selective agonist of $\mathrm{GABA}_{\mathrm{A}}$ receptors containing the $\alpha_{5}$ subunit was applied to the bath saline after similar evoked responses obtained with similar stimulus strength were obtained in both neuronal classes. Figure 4 shows that a similar percentage of the corticostriatal response was enhanced in both neuronal classes during L655-708: ca. $37 \%$ and $35 \%$ for $\mathrm{D}_{1}-\mathrm{SP}+$ and $\mathrm{D}_{2}-\mathrm{ENK}+\mathrm{MSNs}$, respectively $(n=4$ for each MSN type, Figures 4A,B). Histogram in Figure 4C summarizes these results. Thus, although tonic inhibition may serve other purposes (Ade et al., 2008), the present experiments show that it is not the main cause of corticostriatal response differences found between adult $\mathrm{D}_{1}-\mathrm{SP}+$ and $\mathrm{D}_{2}-\mathrm{ENK}+\mathrm{MSNs}$ (Day et al., 2008).

\section{CORTICOSTRIATAL RESPONSES ARE MODIFIED BY DOPAMINE DEPLETION}

As said above, although described differences were discovered in BAC mice, they were corroborated in rats by predicting, based on their electrophysiological responses, which neuron was going to be $\mathrm{SP}+$ or ENK+ by double labeling with biocytin plus immunocytochemistry for the peptide of choice. This prediction showed high accuracy $(P>0.9)$. Neurons that rose doubts coincide with the percentage of neurons that are supposed to co-localize both receptors and peptides (Wang et al., 2007). However, this correspondence needs further corroboration (multiple labeling out of the scope of the present work) by a future study.

In any case, the above experiments allowed us to employ the widely used rat model of Parkinson disease (unilateral lesion with 6-OHDA in the subtantia nigra pars compacta; see Materials and Methods) to compare corticostriatal responses before and after striatal DA depletion. Only lesions that induced $>500$ ipsilateral stereotyped turns ( $n=55$ rats) were considered, to ensure that most dopaminergic innervation in the striatum ipsilateral to the lesion was lost. Further histochemical analysis in a subset of striatal slices confirmed a virtual unilateral loss of tyrosine hydroxylase in lesioned animals. Corticostriatal synaptic responses after DA depletion were therefore examined in slices from PD90 rats. Slices were obtained 2-3 weeks after the lesion and behavioral testing.

In SP+ MSNs (cf., Figures 5A,B), the area under the corticostriatal response was significantly reduced after denervation when compared to the controls: $11,533 \pm 663 \mathrm{mV} \cdot \mathrm{ms}(n=11)$ in the controls vs. $8,769 \pm 527 \mathrm{mV} \cdot \mathrm{ms}(n=16)$ in DA-depleted neurons (Figure 5E; $P<0.025$ ), as well as the half-width: $260 \pm 14 \mathrm{~ms}$ in controls vs. $215 \pm 13 \mathrm{~ms}$ (Figure 5F; $P<0.01$ ).

The opposite was true for ENK+ MSNs (cf., Figures 5C,D), the area under the corticostriatal response was significantly enhanced: cf., $5,828 \pm 736 \mathrm{mV} \cdot \mathrm{ms}(n=9)$ in the controls vs. $8,942 \pm 937 \mathrm{mV} \cdot \mathrm{ms}$ $(n=13)$ in DA-depleted neurons (Figure 5E; $P<0.02)$. The halfwidth also changed: $130 \pm 22 \mathrm{~ms}$ in controls vs. $226 \pm 24 \mathrm{~ms}$ (Figure 5F; $P<0.01$ ). Neither threshold for action potential firing nor the firing frequency nor the passive properties changed as a result of DA depletion. However, a long lasting depolarization predominated during the response of ENK+ MSN after DA depletion. The overall result was that corticostriatal responses from $\mathrm{SP}+$ and ENK+ MSNs after DA depletion looked similar, in contrast to those in the controls (cf., Figures 1 and 5B,D). 
A

$$
D_{1} M S N
$$

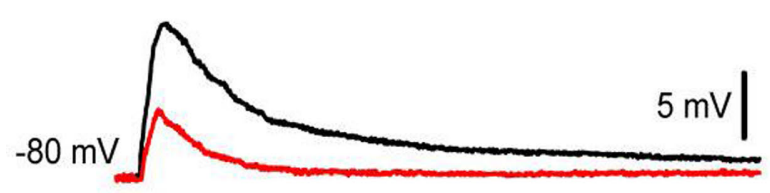

B

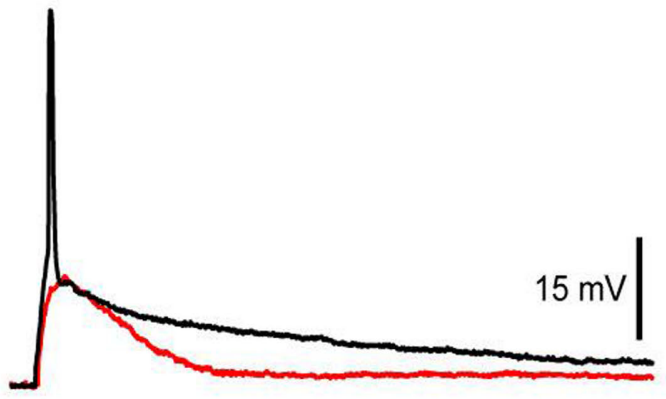

C

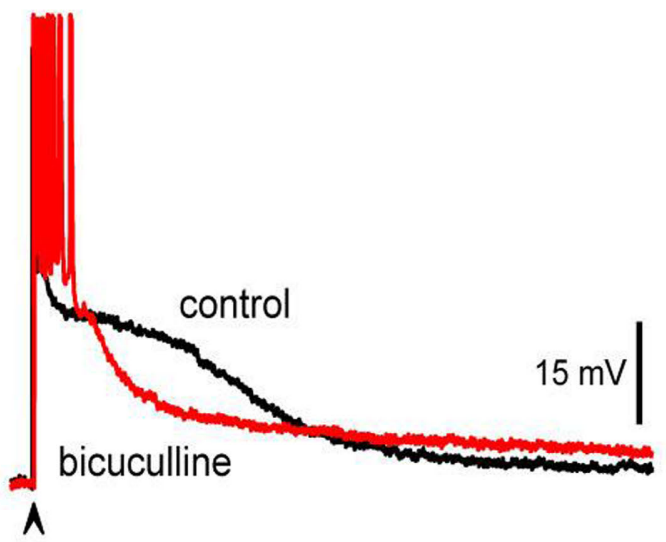

D

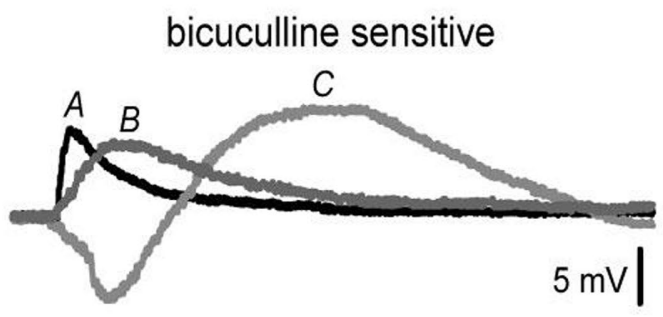

E

$\mathrm{D}_{2} \mathrm{MSN}$

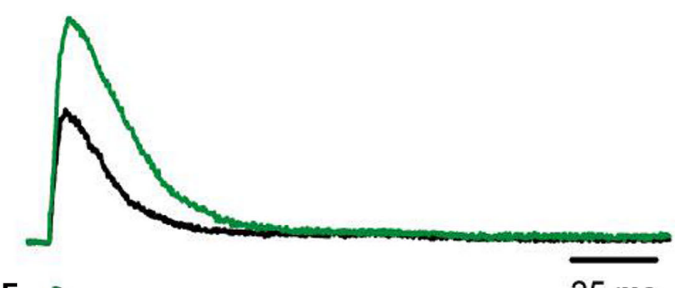

$\mathbf{F}$

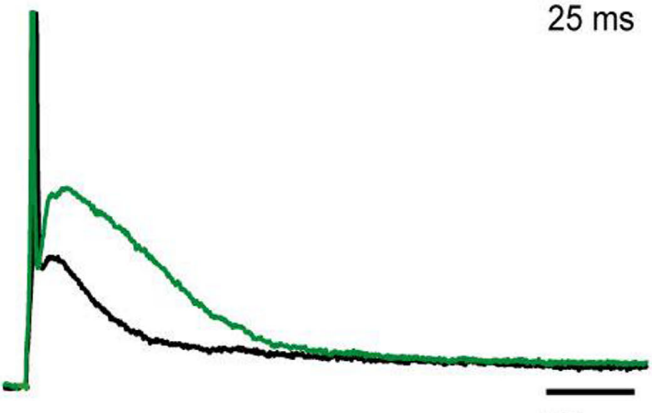

G

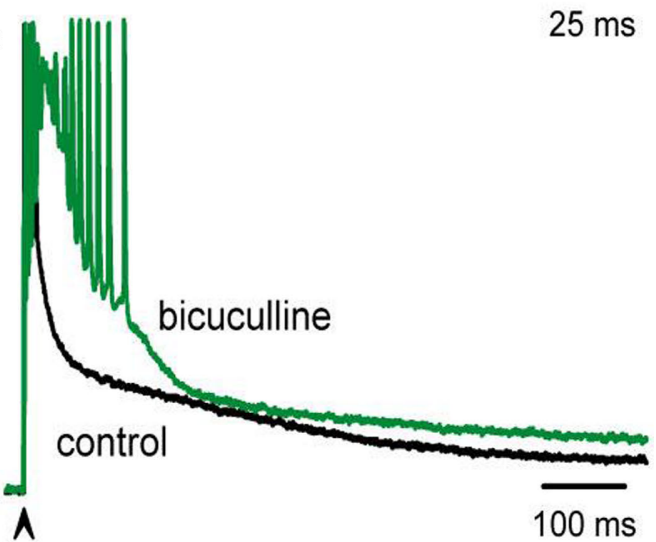

H
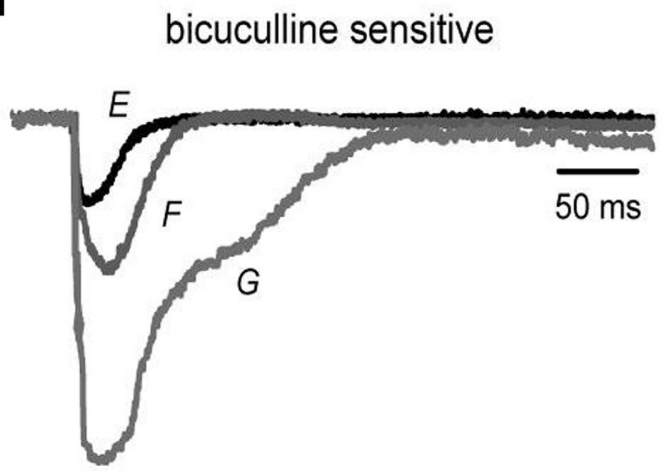

FIGURE 3 | Bicuculline actions differ in $D_{1}$ - and $D_{2}-M S N s$ at different stimulation intensities. (A,E) Blockade of GABAergic synapses during subthreshold synaptic responses $($ black trace $=$ control; colored trace $=$ after bicuculline): both responses are depolarizing and have a similar amplitude in control conditions (about $10 \mathrm{mV}$ ). Notice that GABAergic blockade reduces the synaptic response in $D_{1}$ - and increases the synaptic response in $D_{2}-M S N s$. There is no hyperpolarizing synaptic component in any response in spite of both being bicuculline-sensitive: inhibition is depolarizing in both cases. (B,F) Blockade of GABAergic synapses during threshold synaptic responses. Again, a decrease and an increase in the responses were observed in $D_{1}$ - and $D_{2}-M S N$ s synaptic responses, respectively. Notice that blockade of GABAergic synapses impedes the firing of action potentials in $\mathrm{D}_{1}-\mathrm{MSNs}$ (B) and elicits a local response that distorts synaptic kinetics in $\mathrm{D}_{2}-\mathrm{MSNs}$ (F). (C,G) Blockade of GABAergic synapses in suprathreshold synaptic responses. Superimposed traces cross each other in $D_{1}-M S N s$ and do not cross in $D_{2}-M S N s$. (D-H) Digital subtractions of traces in (A-G): Bicuculline-sensitive component helps in depolarizing all $D_{1}-M S N s$ synaptic responses, except at the beginning of the suprathreshold responses. In contrast, bicuculline enhanced $\mathrm{D}_{2}-\mathrm{MSN}$ s synaptic responses at all stimulus strengths. Inhibition is depolarizing and even excitatory in $D_{1}-M S N s$ whereas it always represses $\mathrm{D}_{2}-\mathrm{MSNs}$ (shunting inhibition). 


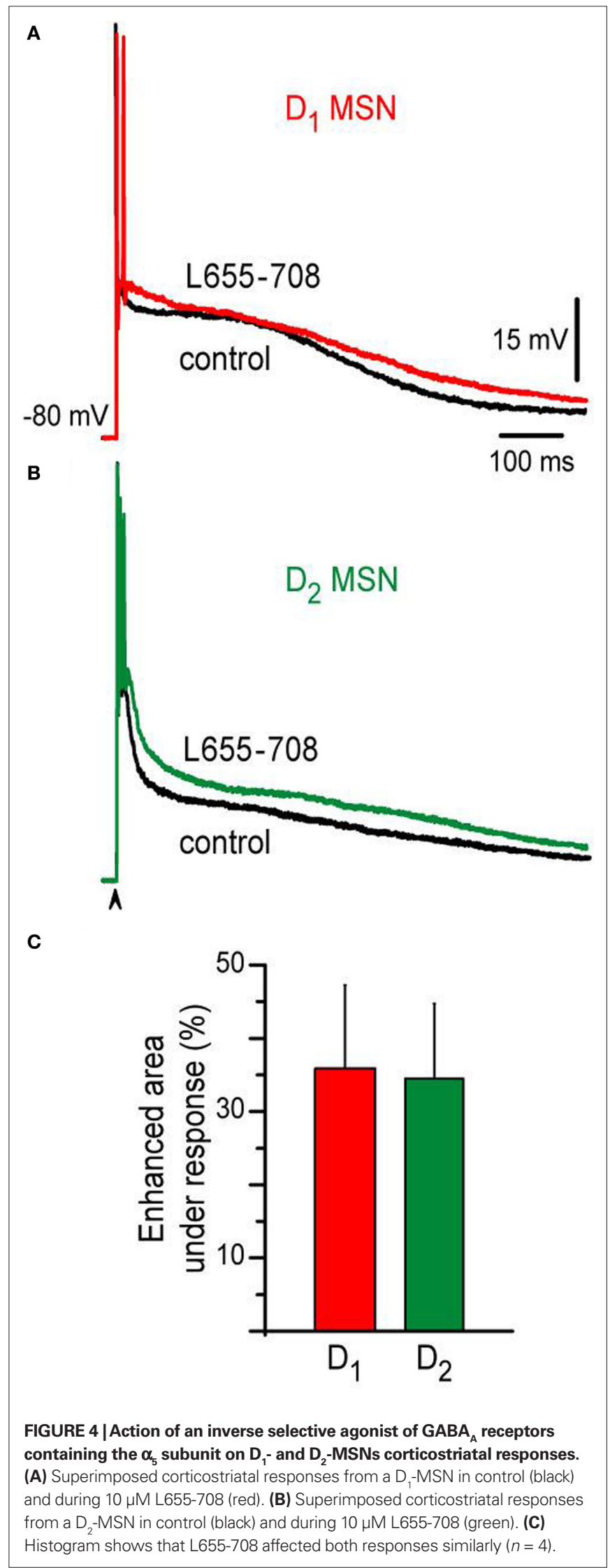

Figure 6 gives a picture of a more thorough comparison at different stimulus strengths (subthreshold, threshold - with spikes clipped - and suprathreshold responses).

Notice that, synaptic potentials were reduced after DA depletion in SP+ MSNs for similar stimulus strengths. DA depletion resulted in smaller corticostriatal responses to any stimulus in direct pathway neurons (Figures 6A-C). Because it was previously shown that inhibition contributes to the depolarizing synaptic response in control neurons (Figure 3), we hypothesized that a decrease in inhibition may be causing these different responses (Taverna et al., 2008; Tecuapetla et al., 2009). In contrast, similar stimulation intensities showed that DA depletion enhanced and prolonged synaptic potentials in ENK+ MSN (Figures 6D-F) even more than in control conditions, resulting in larger corticostriatal responses to any stimulus in indirect pathway neurons (Figures 6D-F), as though inhibition was less capable to control intrinsic excitability in ENK+ MSNs (Taverna et al., 2008; Tecuapetla et al., 2009).

Although no significant differences were observed in the areas under the response for suprathreshold responses (Figure 6I), comparisons below threshold level did show a clear difference: corticostriatal responses are smaller in direct pathway neurons after DA depletion, establishing new evidence of a functional decrease in direct pathway neurons during parkinsonism, and secondly, corticostriatal responses are larger in indirect pathway neurons after DA depletion supporting the long time hypothesized enhancement of indirect pathway function after denervation. Besides rendering this new evidence, the results show that changes suffered after DA depletion resemble those obtained with $\mathrm{GABA}_{\mathrm{A}}$ receptor blockade (Figure 3), in the sense that one response is decreased and the other is increased after the experimental maneuver. Therefore, we decided to compare $\mathrm{GABA}_{\mathrm{A}}$ receptor blockade in direct and indirect neurons after DA depletion.

\section{INHIBITORY CONTRIBUTION TO SUPRATHRESHOLD RESPONSES IS MODIFIED AFTER DA DEPLETION}

$10 \mu \mathrm{M}$ bicuculline were then added while evoking a corticostriatal response in each neuron class subject to DA depletion. Subthreshold synaptic responses behaved in the same way as the controls during $\mathrm{GABA}_{\mathrm{A}}$ receptor blockade: bicuculline reduced these responses in SP+ MSNs and enhanced them in ENK+ MSNs neurons (cf., Figures 7A-G).

However, bicuculline effects were weaker in SP+ MSNs as compared to controls from intact animals (Figure 7Da). In fact, at threshold and suprathreshold intensities responses in direct pathway neurons show less inhibition during the early response $16 \pm 3 \%(n=4)$ (Figure 7Dc vs. $28 \pm 4 \%$ in intact animals; $P<0.03$ ) (Taverna et al., 2008; Tecuapetla et al., 2009). Remarkably, the late depolarizing component that prolonged the plateau depolarization in SP+ MSNs was reduced to $15 \pm 4 \%$ (Figure 7Dc vs. $25 \pm 3 \%$ Figure $3 \mathrm{D}$ in intact animals; $P<0.05$ ) supporting the view that recurrent inhibitory inputs sustain the prolonged depolarization (Flores-Barrera et al., 2009).

On the other hand, bicuculline actions in ENK+ MSNs were greatly enhanced as compared to the controls (Figures 7E-H), so that dendritic excitability seemed greatly increased after the lesion 


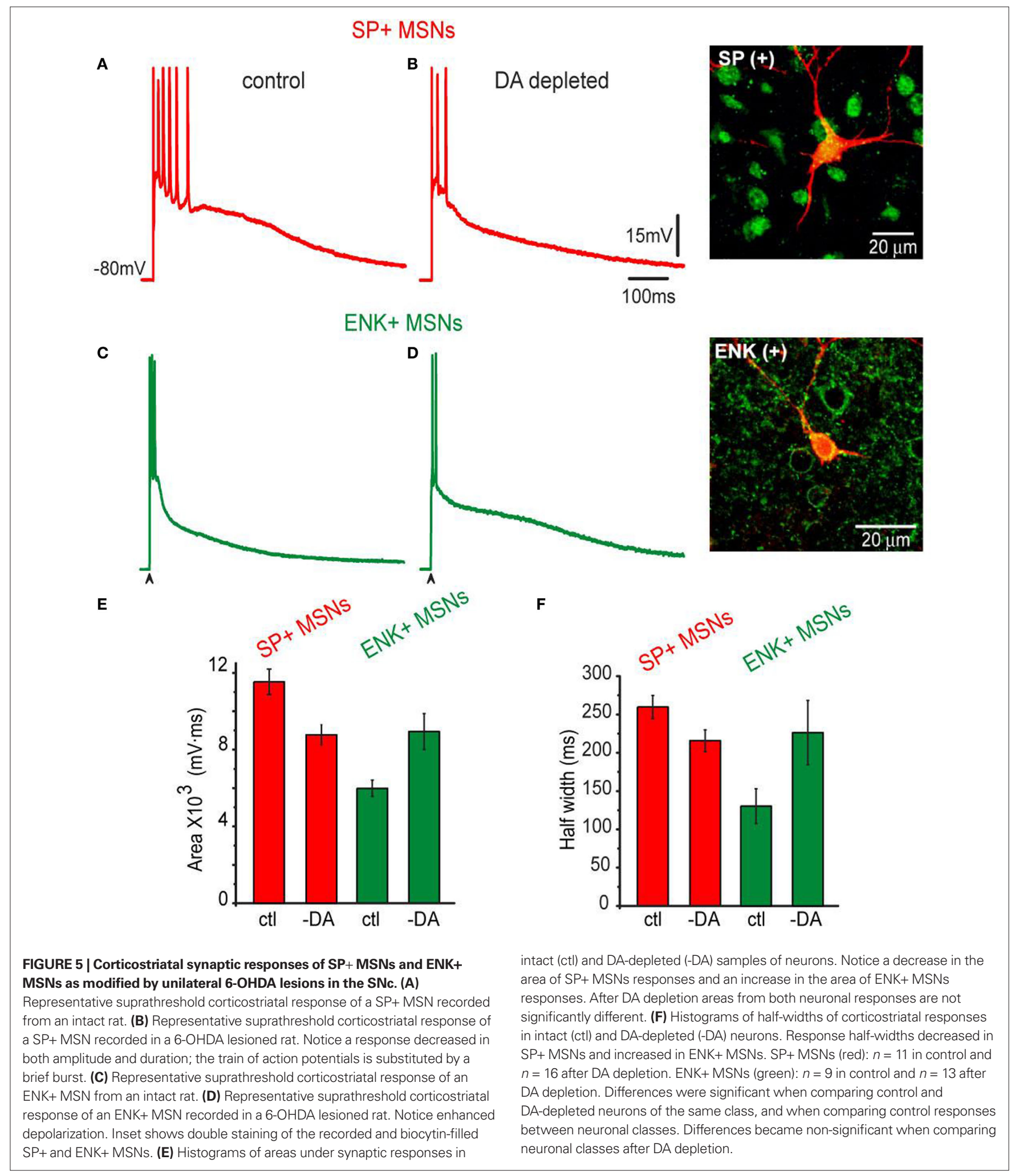

(Day et al., 2008) and intrinsic active components were clear at subthreshold intensities (Figure 7E). The role of inhibition in the suprathreshold response of ENK+ MSNs was also reduced (Taverna et al., 2008; Tecuapetla et al., 2009) and firing was less

repressed: $39 \pm 7 \%$ after the lesion ( $n=5$, vs. $76 \pm 10 \%$ in intact animals: $P<0.01$ ) (Figures 7G,H). No changes in half-widths of the responses were observed after bicuculline (Figures 7C,G, compare Figure $2 \mathrm{H}$ histogram). 


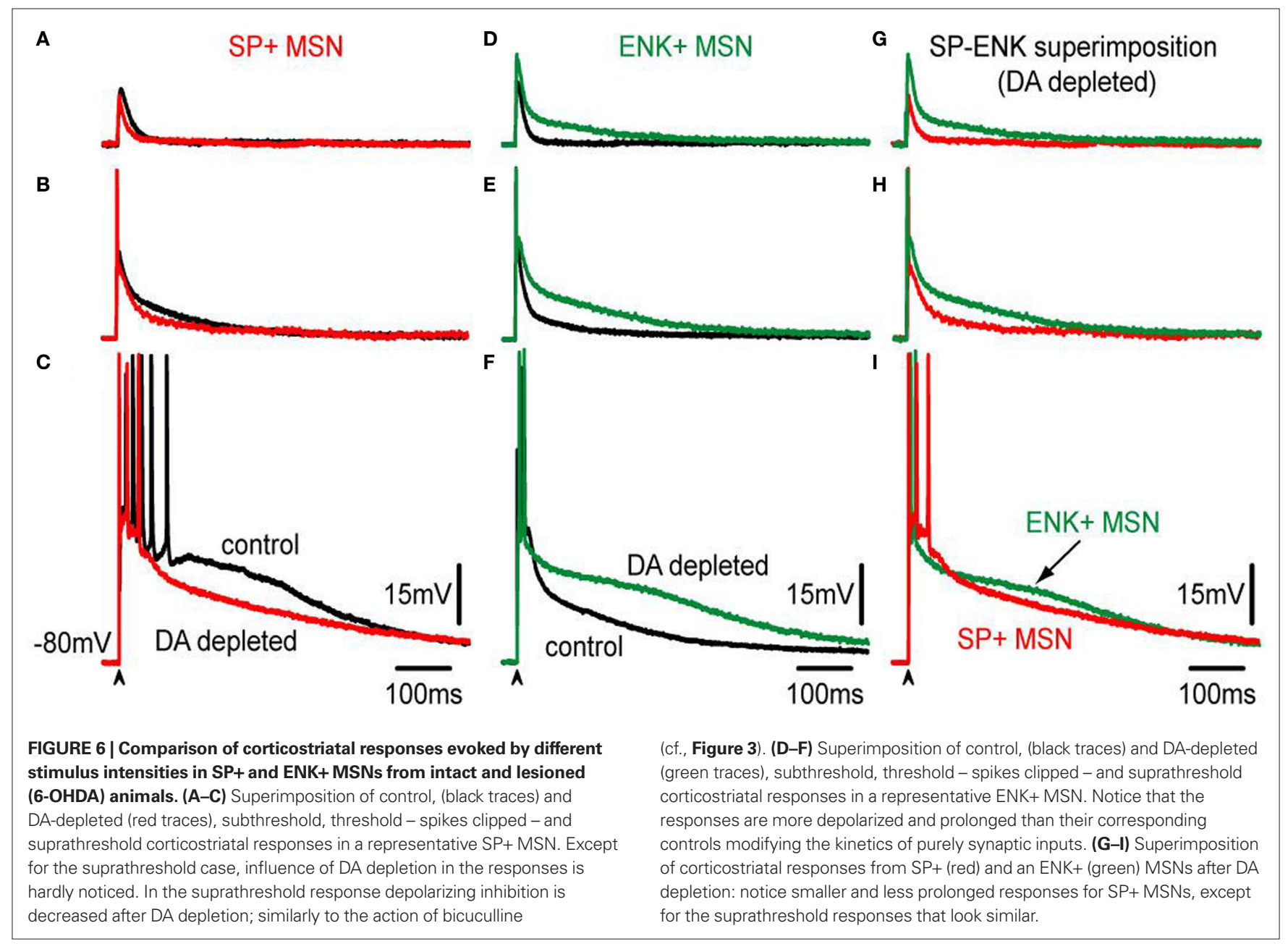

A working hypothesis to explain these data is that a reduction in inhibition is a main cause of the changes observed in the corticostriatal responses in both neuronal classes: most importantly, a decrease in the depolarizing plateau of SP+ MSNs, and a greater intrinsic excitability of ENK+ MSNs dendrites.

\section{DISCUSSION}

This work demonstrates: (1) Corticostriatal responses in direct $\left(\mathrm{D}_{1}-\mathrm{SP}+\right)$ and indirect $\left(\mathrm{D}_{2}-\mathrm{ENK}+\right)$ pathway MSNs are remarkably different and can serve as a footprint to identify the neurons during physiological experiments: they are more prolonged and evoke more action potentials in direct pathway neurons, while an intrinsic autoregenerative response is conspicuous in indirect pathway neurons. (2) A main cause to explain these differences is the role of inhibition: it always depressed $\mathrm{D}_{2}$-ENK+ MSNs responses, whereas it depolarizes or has a dual role in $\mathrm{D}_{1}-\mathrm{SP}+\mathrm{MSN}$ responses, (3) Corticostriatal responses changed dramatically after DA depletion: a reduction in the response was seen in $\mathrm{SP}+-\mathrm{MSN}$ s and an enhancement in the response was seen in ENK+-MSNs. The end result was that their electrophysiological differences appeared less evident after DA depletion. (4) Changes in corticostriatal responses after DA depletion may in part be explained by a decrease of inhibition in both neuronal classes.

\section{CORTICOSTRIATAL RESPONSES IN DIRECT AND INDIRECT MSNS ARE DIFFERENT}

In both neuronal classes a single stimulus to the corticostriatal pathway can induce a slow (plateau-like) orthodromic depolarization with repetitive firing (Bargas et al., 1991; Kita, 1996; Vergara et al., 2003; Tseng et al., 2007; Flores-Barrera et al., 2009). However, corticostriatal responses are more prolonged and evoke more action potentials in $\mathrm{D}_{1}-\mathrm{SP}+\mathrm{MSN}$, whereas an intrinsic autoregenerative response is present in $\mathrm{D}_{2}-\mathrm{ENK}+\mathrm{MSN}$ due to their more patent dendritic excitability (Kreitzer and Malenka, 2007; Shen et al., 2007; Ade et al., 2008; Cepeda et al., 2008; Day et al., 2008; Valjent et al., 2009). This intrinsic response has a role in shaping the response.

Once matched to their eGFP labeled neurons, it became clear that corticostriatal responses from $\mathrm{D}_{1}-\mathrm{SP}+$ and $\mathrm{D}_{2}-\mathrm{ENK}+\mathrm{MSNs}$ are so different that they cannot be confused. Thus, a prediction based on immunocytochemistry for SP and ENK in both rat and mice neurons yielded a high probability in ascertaining the identity of striatal neurons by looking at their corticostriatal responses $(P>0.9)$. Because doubtful cases coincide with the percentage of neurons that may co-localize $\mathrm{D}_{1}$ and $\mathrm{D}_{2}$ receptors it is necessary to perform further studies to fully demonstrate this point. Finally, 
A
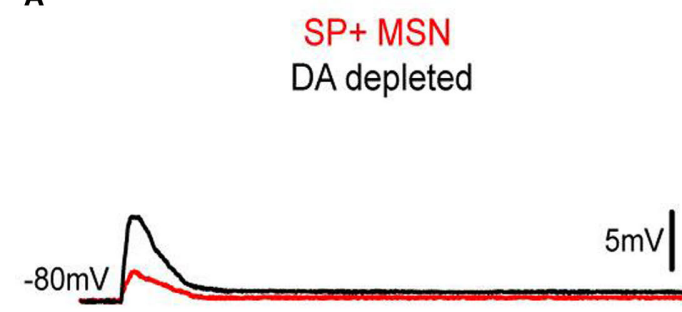

B

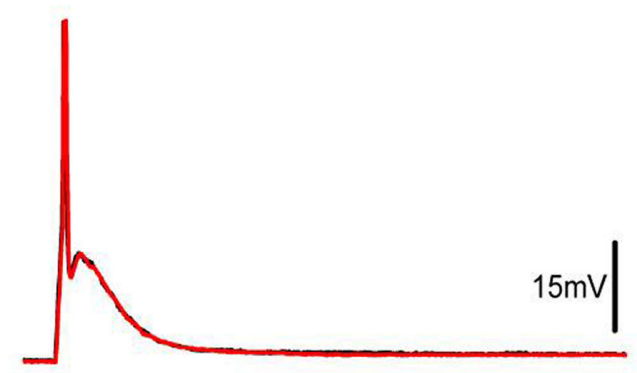

C

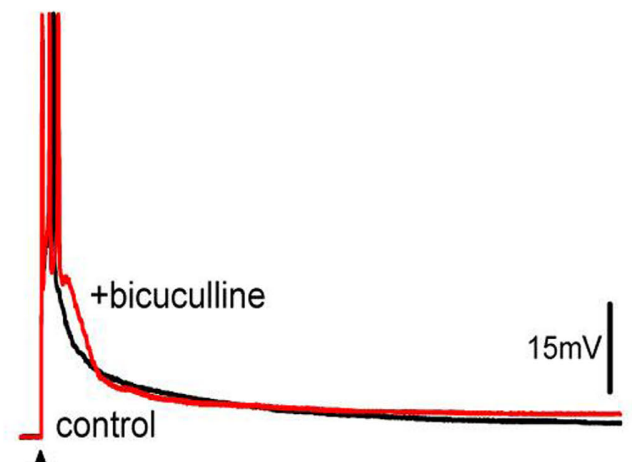

D

bicuculline sensitive

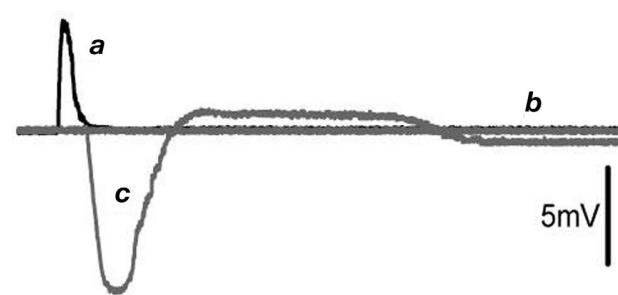

E

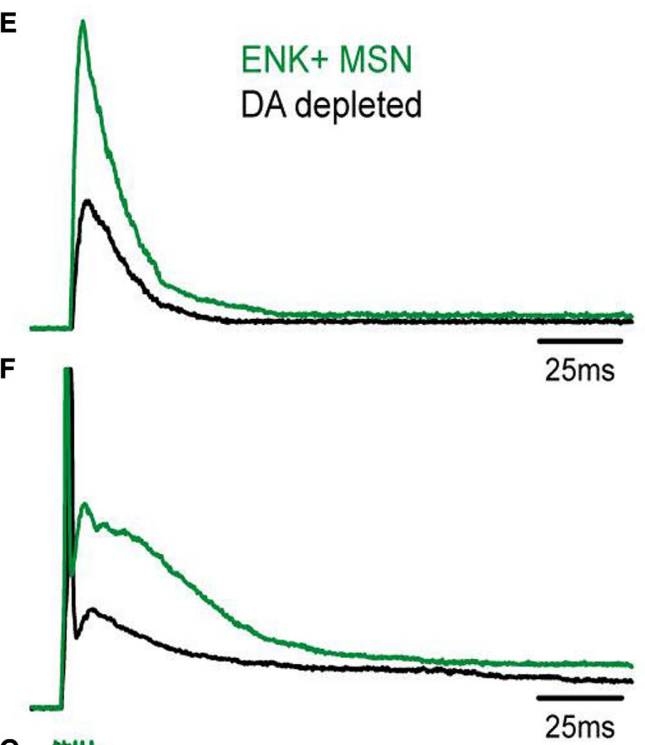

G

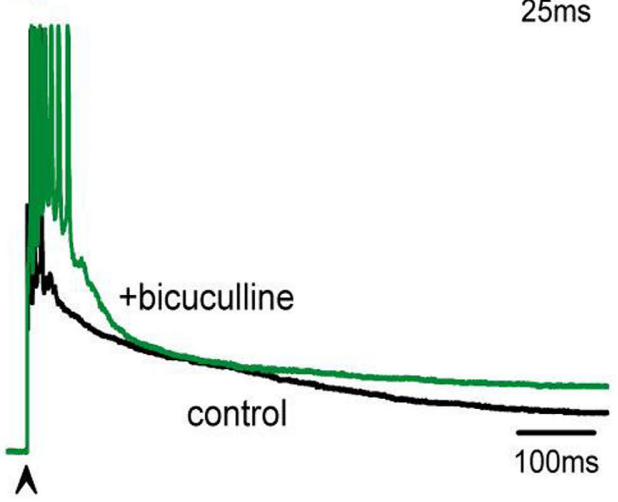

bicuculline sensitive

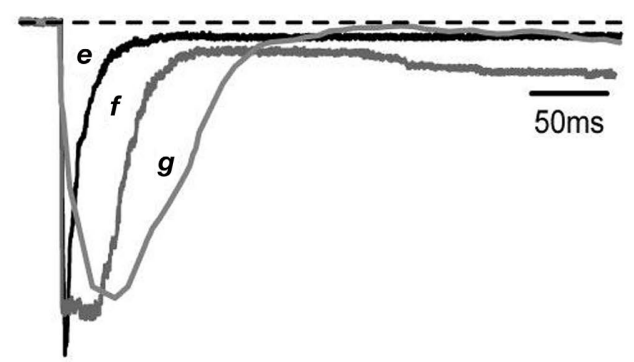

are compared in the same neurons before and during bicuculline (colored traces): a great decrease in bicuculline action is clearly visible in SP+ MSNs (cf., Figure 3), confirming that recruiting of inhibitory inputs is hindered after attaining certain stimulus strengths. A robust depolarization with a prolonged train of spikes is evident in ENK+ MSNs at suprathreshold levels. (D) Digital subtractions of responses in SP+ MSNs (a-c) are signaled by corresponding letters. Notice: initial depolarization due to the inhibitory component is much decreased in subthreshold responses (a), while at threshold level bicuculline action seems occluded or not participating (b). Suprathreshold response reveals inhibition of initial depolarization (c) but an almost complete lack of inhibitory participation in the sustaining of the late depolarizing plateau potential. (H) By comparison, bicuculline-sensitive components subtracted from ENK+ MSNs responses (e-g) have about the same amplitude for all responses, although more prolonged with increases in stimulus strength, suggesting the activation of intrinsic currents. 
it is necessary to find out the class of intrinsic current involved in the autoregenerative response of $\mathrm{D}_{2}$-MSNs, although previous evidence favors $\mathrm{Na}^{+}$- and $\mathrm{Ca}^{2+}$-currents (Bargas et al., 1991; Carter and Sabatini, 2004; Cepeda et al., 2008; Day et al., 2008; CarrilloReid et al., 2009b).

\section{DIFFERENT ROLES OF INHIBITION IN THE CORTICOSTRIATAL RESPONSES OF DIRECT AND INDIRECT MSNs}

Different cortical inputs to each class of MSNs (Ingham et al., 1998; Lei et al., 2004; Mallet et al., 2006; Cepeda et al., 2008) may in part explain some of the differences described. However, the present results demonstrate that the role of intrastriatal inhibition on the corticostriatal responses of these neuronal classes is radically different and could explain a part of the variation. Reversal inhibitory potential is similar and above the resting potential in all MSNs recorded by numerous studies using different techniques including gramicidin patches (Misgeld et al., 1982; Jiang and North, 1991; Bennett and Bolam, 1994; Kita, 1996; Ramanathan et al., 2002; Koos et al., 2004; Bracci and Panzeri, 2006; Dehorter et al., 2009; FloresBarrera et al., 2009); making it hard to think in a bias towards one class of neuron given that each class is about $50 \%$ of the population. In addition, no hyperpolarizing potential has been reported, or was observed in this study, when evoking synaptic responses of different intensities at near $-80 \mathrm{mV}$ resting potential, even when all responses from each class of neuron had a bicuculline-sensitive component. Therefore, a depolarizing inhibition is present in both responses. Nevertheless, inhibition always depressed the response in $\mathrm{D}_{2}$-ENK+ MSNs, whatever the stimulus strength, whereas it helped to depolarize the response in $\mathrm{D}_{1}-\mathrm{SP}+\mathrm{MSN}$, thus making a radical distinction between the actions of $\mathrm{GABA}_{\mathrm{A}}$ receptors in each neuronal class. Moreover, because recordings were done with sharp electrodes without internal chloride solution, significant differences between neuronal populations cannot be attributed to changes in intracellular media. Thus, a question arises about the reasons of such a different inhibitory role in each neuronal class.

Bicuculline actions in $\mathrm{D}_{1}-\mathrm{SP}+\mathrm{MSN}$ are easier to explain: Since inhibition is depolarizing and contributes to evoked synaptic depolarization from the cortex, blockade of inhibition leads to a decrease in synaptic depolarization. Two peculiar properties of this inhibitory depolarization are worth mentioning: first, the fact that it facilitates the firing of action potentials during threshold responses (excitatory depolarizing inhibition), and secondly, its dual action at suprathreshold levels: restraining firing at the beginning of the response and supporting the prolonged depolarization during the plateau (Gulledge and Stuart, 2003; Bartos et al., 2007; Mann and Paulsen, 2007; Flores-Barrera et al., 2009). In fact, a previous experimental and modeling study by our group simulated initial shunting inhibition with strong somatic inhibitory inputs, and later depolarization with recurrent reverberant and depolarizing inhibitory inputs along the dendrites (Flores-Barrera et al., 2009). Recurrent architecture in the striatal microcircuit facilitates prolonged depolarizations and explains spontaneous synchronization and micro-circuitry activity, which are greatly altered when inhibition is reduced (Carrillo-Reid et al., 2008, 2009a).

Bicuculline actions in $\mathrm{D}_{2}$-ENK+ MSNs can be explained by the distinct and higher dendritic excitability present in these neurons (Galarraga et al., 1997; Day et al., 2008): even when inhibition is also depolarizing, it has the role of restraining stronger depolarizing intrinsic currents (shunting inhibition) (Bartos et al., 2007). Thus, blockade of inhibition in these neurons leads to a disclosure of intrinsic currents which at subthreshold and threshold levels may consist in local non-propagated responses that enhance and alter the kinetics of synaptic responses, but at suprathreshold levels become autoregenerative, propagated responses. In fact, these responses underlie the brief train of action potentials that these neurons display. If uncontrolled by inhibition, these intrinsic responses may increase the duration of action potential trains, although many of these spikes undergo inactivation. The ionic nature of these intrinsic responses is currently under investigation, and besides inhibition, they probably need $\mathrm{K}^{+}$currents to be controlled (Day et al., 2008).

\section{CORTICOSTRIATAL RESPONSES AFTER DA DEPLETION REFLECT A LOSS IN INHIBITION}

Striatal DA depletion produced a reduction in amplitude and duration of corticostriatal responses in SP+-MSNs and an enhancement of duration and amplitude in ENK+ -MSNs, basically, similar changes than those produced by $\mathrm{GABA}_{\mathrm{A}}$ receptor blockade (see above). Accordingly, a working hypothesis to explain these changes is that a decrease in inhibition resulted from DA depletion (Taverna et al., 2008; Tecuapetla et al., 2009). And in fact, altered bicuculline actions in both responses supported a decrease in inhibition as a main physiopathological change during parkinsonism.

The end result was that both responses lost their conspicuous electrophysiological differences in shape after DA depletion. In control conditions direct and indirect pathways are not "balanced" in the sense that both show a similar response to cortical commands. On the contrary, duration of $\mathrm{D}_{1}-\mathrm{SP}+\mathrm{MSN}$ responses is larger than that in $\mathrm{D}_{2}$-ENK+ MSNs (see above). A decrease in inhibition leads to a decrease in the response of $\mathrm{D}_{1}-\mathrm{SP}+\mathrm{MSN}$ sustained in part by recurrent inhibition (see above). This result adds to the body of evidence that implies a decrease in direct pathway function during parkinsonism (DeLong, 1990; Obeso et al., 2004). By contrast, a decrease in inhibition leads to an increase in the escape of intrinsic depolarizing currents in $\mathrm{D}_{2}$-ENK+ neurons thus enhancing their corticostriatal response. This outcome supports the hypothesis that indirect pathway neurons have an increased excitability during parkinsonism (DeLong, 1990; Obeso et al., 2004; Day et al., 2008). These results give a functional context to our findings, but paradoxically, direct and indirect pathways now appear to be "balanced" with respect to their corticostriatal response shape.

However, this apparent similarity between response shapes hides significantly different mechanism of integration to generate the responses (see above). A more detailed analysis shows that in fact, $\mathrm{D}_{2}-\mathrm{ENK}+$ neurons are more excitable than $\mathrm{D}_{1}-\mathrm{SP}+$ neurons both in control and after DA depletion. Dopaminergic denervation actually worsens this disparity. In addition, recurrent inhibition between spiny neurons is not similar between direct and indirect pathway neurons (Tecuapetla et al., 2009), direct connections being enhanced whereas indirect connections are being depressed by dopamine (Guzman et al., 2003; Tecuapetla et al., 2007, 2009). Logically, denervation will change this relation affecting the connections within a pathway and among the pathways, altering circuit dynamics (Carrillo-Reid et al., 2008, 2009a,b). Moreover, although a different contribution of inhibition provided by fast-spiking (FS) 
interneurons is now under debate (Salin et al., 2009; Gittis et al., 2010; Planert et al., 2010), clearly, there is a potentiation in synaptic inhibition provided by low threshold spiking (LTS) interneurons (Dehorter et al., 2009) after DA depletion. Given the role of these different interneuron classes in the circuit (Whittington and Traub, 2003), these changes would diminish prokinetic fast rhythms and promote akinetic slower rhythms (Hutchinson et al., 2004). Therefore, further investigation is needed to see if these alterations are reflected in the corticostriatal responses.

\section{REFERENCES}

Ade, K. K., Janssen, M. J., Ortinski, P. I., and Vicini, S. (2008). Differential tonic GABA conductances in striatal medium spiny neurons. J. Neurosci. 28, 1185-1197.

Bargas, J., Galarraga, E., and Aceves, J. (1991). Dendritic activity on neostriatal neurons as inferred from somatic intracellular recordings. Brain Res. 539, 159-163.

Bartos, M., Vida, I., and Jonas, P. (2007). Synaptic mechanisms of synchronized gamma oscillations in inhibitory interneuron networks. Nat. Rev. Neurosci. 8, 45-56.

Bennett, B. D., and Bolam, J. P. (1994). Synaptic input and output of parvalbumin-immunoreactive neurons in the neostriatum of the rat. Neuroscience 62, 707-719.

Bracci, E., and Panzeri, S. (2006). Excitatory GABAergic effects in striatal projection neurons. J. Neurophysiol. 95, 1285-1290.

Carrillo-Reid, L., Tecuapetla, F., IbañezSandoval, O., Hernandez-Cruz, A., Galarraga, E., and Bargas, J. (2009a). Activation of the cholinergic system endows compositional properties to striatal cell assemblies. J. Neurophysiol. 101, 737-749.

Carrillo-Reid, L., Tecuapetla, F., Vautrelle, N., Hernández, A., Vergara, R., Galarraga, E., and Bargas, J. (2009b). Muscarinic enhancement of persistent sodium current synchronizes striatal medium spiny neurons. $J$. Neurophysiol. 102, 682-690.

Carrillo-Reid, L., Tecuapetla, F., Tapia, D., Hernandez-Cruz, A., Galarraga, E., Drucker-Colin, R., and Bargas, J. (2008). Encoding network states by striatal cell assemblies. J. Neurophysiol. 99, 1435-1450.

Carter, A. G., Sabatini, B. L. (2004). Statedependent calcium signaling in dendritic spines of striatal medium spiny neurons. Neuron 44, 483-493.

Cepeda, C., Andre, V. M., Yamazaki, I., Wu, N., Kleiman-Weiner, M., and Levine, M.S. (2008). Differential electrophysiological properties of dopamine D1 and D2 receptor-containing striatal medium-sized spiny neurons. Eur. J. Neurosci. 27, 671-682.
Day, M., Wokosin, D., Plotkin, J.L., Tian,X., and Surmeier, D. J. (2008). Differential excitability and modulation of striatal medium spiny neuron dendrites. J. Neurosci. 28, 11603-11614.

Dehorter, N., Guigoni, C., Lopez, C., Hirsch, J., Eusebio, A., Ben-Ari, Y., and Hammond, C. (2009). Dopaminedeprived striatal GABAergic interneurons burst and generate repetitive gigantic IPSCs in medium spiny neurons. J. Neurosci. 29, 7776-7787.

DeLong, M. R. (1990). Primate models of movement disorders of basal ganglia origin. Trends Neurosci. 13, 281-285.

Dunnett, S. B., Bjorklund, A., Stenevi, U., and Iversen, S. D. (1981). Behavioural recovery following transplantation of substantia nigra in rats subjected to 6-OHDA lesions of the nigrostriatal pathway. I. Unilateral lesions. Brain Res. 215, 147-161.

Flores-Barrera, E., Laville, A., Plata, V., Tapia, D., Bargas, J., and Galarraga, E. (2009). Inhibitory contribution to suprathreshold corticostriatal responses: an experimental and modeling study. Cell. Mol. Neurobiol. 29, 719-731.

Galarraga, E., Hernandez-Lopez, S., Reyes, A., Barral, J., and Bargas, J. (1997). Dopamine facilitates striatal EPSPs through an L-type $\mathrm{Ca}^{2+}$ conductance. Neuroreport 8, 2183-2186.

Gerfen, C. R., and Young, W. S. III (1988). Distribution of striatonigral and striatopallidal peptidergic neurons in both patch and matrix compartments: an in situ hybridization histochemistry and fluorescent retrograde tracing study. Brain Res. 460, 161-167.

Gertler, T. S., Chan, C. S., and Surmeier, D. J. (2008). Dichotomous anatomical properties of adult striatal medium spiny neurons. J. Neurosci. 28, 10814-10824.

Gittis, A. H., Nelson, A. B., Thwin, M. T., Palop, J. J., and Kreitzer, A. C. (2010). Distinct roles of GABAergic interneurons in the regulation of striatal output pathways. J. Neurosci. 30, 2223-2234.

Gruber, A. J., Solla, S. A., Surmeier, D. J., and Houk, J. C. (2003). Modulation of striatal single units by expected reward: a spiny neuron model displaying

\section{ACKNOWLEDGMENTS}

We thank Antonio Laville and Marcela Palomero-Rivero for technical support and advice and to Dr. Claudia Rivera for animal care. This work was supported by grants from a Project Program grant IMPULSA 03 from UNAM, by Consejo Nacional de Ciencia y Tecnologia (Mexico) Grant 98004, and by grants from Direccion General de Asuntos del Personal Academico. Universidad Nacional Autonoma de Mexico: IN205610 and IN206010 to Bargas J and Galarraga E.

dopamine-induced bistability. $J$. Neurophysiol. 90, 1095-1114.

Gulledge, A. T., and Stuart, G. J. (2003). Excitatory actions of GABA in the cortex. Neuron 37, 299-309.

Gustafson, N., Gireesh-Dharmaraj, E., Czubayko, U., Blackwell, K. T., and Plenz, D. A. (2006). Comparative voltage and current-clamp analysis of feedback and feedforward synaptic transmission in the striatal microcircuit in vitro. J. Neurophysiol. 95, 737-752.

Guzman, J. N., Hernandez, A., Galarraga, E., Tapia, D., Laville, A., Vergara, R., Aceves, J., and Bargas, J. (2003). Dopaminergic modulation of axon collaterals interconnecting spiny neurons of the rat striatum. J. Neurosci. 23, 8931-8940.

Hutchinson, W. D., Dostrovsky, J. O., Walters, J. R., Courtemanche, R., Boraud, T., Goldberg, J., and Brown, P. (2004). Neuronal oscillations in the basal ganglia and movement disorders: evidence from whole animal and human recordings. J. Neurosci. 24, 9240-9243.

Inaji, M., Okauchi, T., Ando, K., Maeda, J., Nagai, Y., Yoshizaki, T., Okano, H., Nariai, T., Ohno, K., Obayashi, S., Higuchi, M., and Suhara, T. (2005). Correlation between quantitative imaging and behavior in unilaterally 6-OHDA-lesioned rats. Brain Res. 1064, 136-145.

Ingham, C. A., Hodd, S. H., Taggart, P., and Arbuthnott, G. W. (1998). Plasticity of synapses in the rat neostriatum after unilateral lesion of the nigrostriatal dopaminergic pathway. J. Neurosci. 18, 4732-4743.

Jiang, Z. G., and North, R. A. (1991). Membrane properties and synaptic responses of rat striatal neurons in vitro. J. Physiol. 443, 533-553.

Kawaguchi, Y., Wilson, C. J., Augood, S. J., and Emson, P. C. (1995). Striatal interneurons: chemical, physiological and morphological characterization. Trends Neurosci. 18, 527-535.

Kita, H. (1996). Glutamatergic and GABAergic postsynaptic responses of striatal spiny neurons to intrastriatal and cortical stimulation recorded in slice preparations. Neuroscience 70 , 925-940.
Koos, T., Tepper, J. M., and Wilson, C. J. (2004). Comparison of IPSCs evoked by spiny and fast-spiking neurons in the neostriatum. J. Neurosci. 24, 7916-7922.

Kreitzer,A. C., and Malenka, R. C. (2007). Endocannabinoid-mediated rescue of striatal LTD and motor deficits in Parkinson's disease models. Nature 445, 643-647.

Lei, W., Jiao, Y., Del Mar, N., and Reiner, A. (2004). Evidence for differential cortical input to direct pathway versus indirect pathway striatal projection neurons in rats. J. Neurosci. 24, 8289-8299.

Lemus-Aguilar, I., Bargas, J., Tecuapetla, F., Galarraga, E., Carrillo-Reid, L. (2006) Diseño modular de instrumentacion virtual para la manipulacion y el análisis de señales electrofisiológicas. Rev. Mex. Ing. Biomed. 27, 82-92.

Mallet, N., Ballion, B., Le Moine, C., and Gonon, F. (2006). Cortical inputs and GABA interneurons imbalance projection neurons in the striatum of Parkinsonian rats. J. Neurosci. 26, 3875-3884.

Mann, E. O., and Paulsen, O. (2007). Role of GABAergic inhibition in hippocampal network oscillations. Trends Neurosci. 30, 343-349.

Misgeld, U., Wagner, A., and Ohno, T. (1982). Depolarizing IPSPs and depolarization by GABA of rat neostriatum cells in vitro. Exp. Brain Res. 45, 108-114.

Obeso,J.A., Rodriguez-Oroz,M., Marin, C., Alonso, F., Zamarbide, I., Lanciego, J. L. and Rodriguez-Diaz,M.(2004). Theorigin of motor fluctuations in Parkinson's disease: importance of dopaminergic innervation and basal ganglia circuits. Neurology 62, S17-S30.

O’Donnell, P. (2003). Dopamine gating of forebrain neural ensembles. Eur. J. Neurosci. 17, 429-435.

Planert, H., Szydlowski, S. N., Hjorth, J.J.J., Grillner, S., and Silberberg, G. (2010). Dynamics of synaptic transmission between fast-spiking interneurons and striatal projection neurons of the direct and indirect pathways. $J$. Neurosci. 30, 3499-3507.

Ramanathan, S., Hanley, J. J., Deniau, J. M., and Bolam, J. P. (2002). 
Synaptic convergence of motor and somatosensory cortical afferents onto GABAergic interneurons in the rat striatum. J. Neurosci. 22, 8158-8169.

Reyes, A., Galarraga, E., Flores-Hernández, J., Tapia, D., and Bargas, J. (1998). Passive properties of neostriatal neurons during potassium conductance blockade. Exp. Brain Res. 120, 70-84.

Salin, P., López, I. P., Kachidian, P., BarrosoChinea, P., Rico, A. J., Gómez-Bautista, V., Coulon, P., Kerkerian-Le., Goff, L., and Lanciego, J. L. (2009). Changes to interneuron-driven striatal microcircuits in a rat model of Parkinson's disease. Neurobiol. Dis. 34, 545-552.

Shen, W., Tian, X., Day, M., Ulrich, S., Tkatch, T., Nathanson, N. M., and Surmeier, D. J. (2007). Cholinergic modulation of Kir2 channels selectively elevates dendritic excitability in striatopallidal neurons. Nat. Neurosci. 10, 1458-1466.

Taverna, S., Ilijic, E., and Surmeier, D. J. (2008). Recurrent collateral connections of striatal medium spiny neurons are disrupted in models of Parkinson's Disease. J. Neurosci. 28, 5504-5512.

Tecuapetla, F., Carrillo-Reid, L., Bargas, J., and Galarraga, E. (2007).
Dopaminergic modulation of short term synapitc plasticity at striatal inhibitory synapses. Proc. Natl. Acad. Sci. USA. 104, 10258-10263.

Tecuapetla, F., Carrillo-Reid, L., Guzman, J. N., Galarraga, E., and Bargas, J. (2005). Different inhibitory inputs onto neostriatal projection neurons as revealed by field stimulation. $J$. Neurophysiol. 93, 1119-1126.

Tecuapetla, F., Koós, T., Tepper, J. M., Kabbani, N., and Yeckel, M. F. (2009). Differential dopaminergic modulation of neostriatal synaptic connections of striatopallidal axon collaterals. J. Neurosci. 29, 8977-8990.

Tseng, K. Y., Snyder-Keller, A., and O’Donnell, P. (2007). Dopaminergic modulation of striatal plateau depolarizations in corticostriatal organotypic cocultures. Psychopharmacology 191, 627-640.

Tunstall, M. J., Oorschot, D. E., Kean, A., and Wickens, J. R. (2002). Inhibitory interactions between spiny projection neurons in the rat striatum. J. Neurophysiol. 88, 1263-1269.

Valjent, E., Bertran-Gonzalez, J., Hervé, D., Fisone, G., and Girault, J.A. (2009). Looking BAC at striatal signaling: cell-specific analysis in new transgenic mice. Trends Neurosci. 32, 538-547.

Vergara, R., Rick, C., Hernandez-Lopez, S., Laville, J.A., Guzman, J. N., Galarraga, E., Surmeier, D. J., and Bargas, J. (2003). Spontaneous voltage oscillations in striatal projection neurons in a rat corticostriatal slice. J. Physiol. 553, 169-182.

Vida, I., Bartos, M., and Jonas, P. (2006). Shunting inhibition improves robustness of gamma oscillations in hippocampal interneuron networks by homogenizing firing rates. Neuron 49, 107-117.

Wang, H. B., Deng, Y. P., and Reiner, A. (2007). In situ hybridization histochemical and immunohistochemical evidence that striatal projection neurons co-containing substance $\mathrm{P}$ and enkephalin are overrepresented in the striosomal compartment of striatum in rats. Neurosci. Lett. 425, 195-199.

Whittington, M. A., and Traub, R. D. (2003). Interneuron diversity series: inhibitory interneurons and network oscillations in vitro. Trends Neurosci. 26, 676-682.

Wilson, C. J. (1986). Postsynaptic potentials evoked in spiny neostriatal projection neurons by stimulation of ipsilateral and contralateral neocortex. Brain Res. 367, 201-213.

Conflict of Interest Statement: The authors declare that the research was conducted in the absence of any commercial or financial relationships that could be construed as a potential conflict of interest.

Received: 10 February 2010; paper pending published: 27 March 2010; accepted: 10 May 2010; published online: 10 June 2010.

Citation: Flores-Barrera E, VizcarraChacón BJ, Tapia D, Bargas Jand Galarraga E (2010) Different corticostriatal integration in spiny projection neurons from direct and indirectpathways. Front. Syst. Neurosci. 4:15. doi: 10.3389/fnsys.2010.00015 Copyright (C) 2010 Flores-Barrera, VizcarraChacón, Tapia, Bargas and Galarraga. This is an open-access article subject to an exclusive license agreement between the authors and the Frontiers Research Foundation, which permits unrestricted use, distribution, and reproduction in any medium, provided the original authors and source are credited. 